\title{
Assessing gray matter volume in patients with idiopathic rapid eye movement sleep behavior disorder
}

\author{
Xian-Hua Han ${ }^{1, \#}$, Xiu-Ming Li ${ }^{1, \#}$, Wei-Jun Tang ${ }^{2}$, Huan $\mathrm{Yu}^{3}$, Ping Wu ${ }^{1}$, Jing-Jie Ge ${ }^{1}$, Jian Wang ${ }^{3}$, Chuan-Tao Zuo ${ }^{1, ~ *}, K$ Kuang-Yu Shi ${ }^{4}$ \\ 1 PET Center, Huashan Hospital, Fudan University, Shanghai, China \\ 2 Department of Radiology, Huashan Hospital, Fudan University, Shanghai, China \\ 3 Department of Neurology, Huashan Hospital, Fudan University, Shanghai, China \\ 4 Department of Nuclear Medicine, Technical University Munich, Munich, Germany
}

Funding: This study was supported by the China-US Biomedical Collaborative Research Program, No. 81361120393 (to CTZ); the National Natural Science Foundation of China, No. 81401135 (to PW), 81671239 (to CTZ); and the Shanghai Sailing Program, No. 18 YF1403100 (to JJG).

\section{Graphical Abstract}

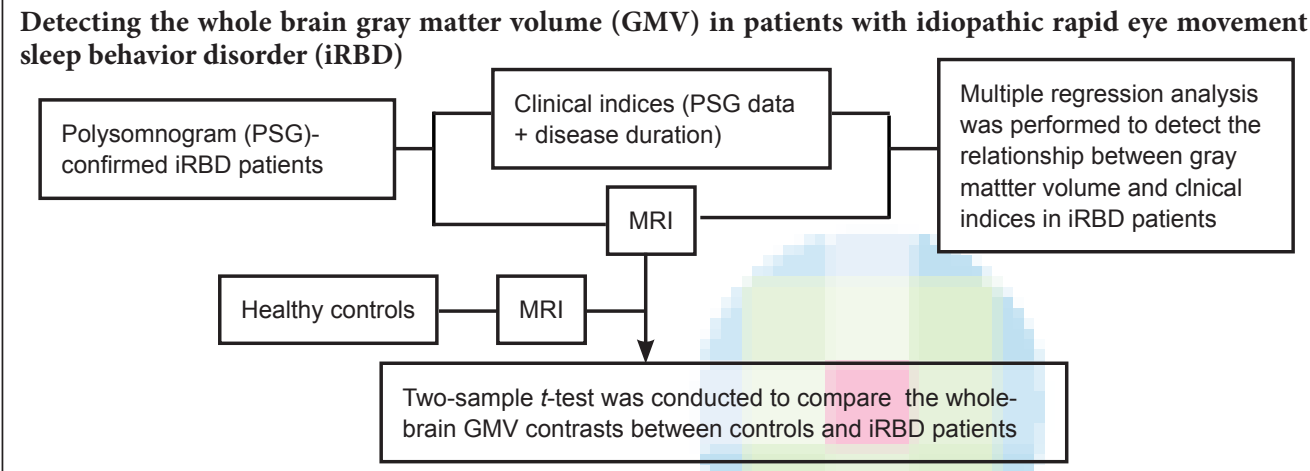

\author{
*Correspondence to: \\ Chuan-Tao Zuo, MD, \\ zuoct_cn2000@126.com \\ \#These authors contributed \\ equally to this study. \\ orcid: \\ 0000-0002-8856-7217 \\ (Chuan-Tao Zuo)
}

doi: $10.4103 / 1673-5374.249235$

Received: July 20, 2018

Accepted: September 18, 2018

\begin{abstract}
Idiopathic rapid eye movement sleep behavior disorder (iRBD) is often a precursor to neurodegenerative disease. However, voxel-based morphological studies evaluating structural abnormalities in the brains of iRBD patients are relatively rare. This study aimed to explore cerebral structural alterations using magnetic resonance imaging and to determine their association with clinical parameters in iRBD patients. Brain structural T1-weighted MRI scans were acquired from 19 polysomnogram-confirmed iRBD patients (male:female 16:3; mean age 66.6 \pm 7.0 years) and 20 age-matched healthy controls (male:female 5:15; mean age $63.7 \pm 5.9$ years). Gray matter volume (GMV) data were analyzed based on Statistical Parametric Mapping 8, using a voxel-based morphometry method and two-sample $t$-test and multiple regression analysis. Compared with controls, iRBD patients had increased GMV in the middle temporal gyrus and cerebellar posterior lobe, but decreased GMV in the Rolandic operculum, postcentral gyrus, insular lobe, cingulate gyrus, precuneus, rectus gyrus, and superior frontal gyrus. iRBD duration was positively correlated with GMV in the precuneus, cuneus, superior parietal gyrus, postcentral gyrus, posterior cingulate gyrus, hippocampus, lingual gyrus, middle occipital gyrus, middle temporal gyrus, and cerebellum posterior lobe. Furthermore, phasic chin electromyographic activity was positively correlated with GMV in the hippocampus, precuneus, fusiform gyrus, precentral gyrus, superior frontal gyrus, cuneus, inferior parietal lobule, angular gyrus, superior parietal gyrus, paracentral lobule, and cerebellar posterior lobe. There were no significant negative correlations of brain GMV with disease duration or electromyographic activity in iRBD patients. These findings expand the spectrum of known gray matter modifications in iRBD patients and provide evidence of a correlation between brain dysfunction and clinical manifestations in such patients. The protocol was approved by the Ethics Committee of Huashan Hospital (approval No. KY2013-336) on January 6, 2014. This trial was registered in the ISRCTN registry (ISRCTN18238599).
\end{abstract}

Key Words: nerve regeneration; idiopathic rapid eye movement sleep behavior disorder; synucleinopathies; magnetic resonance imaging; gray matter volume; statistic parametric mapping; voxel-based morphometry; structure; Parkinson's disease; neurodegenerative diseases; neural regeneration

Chinese Library Classification No. R445; R741

\section{Introduction}

Idiopathic rapid eye movement (REM) sleep behavior disorder (iRBD) is a parasomnia characterized by the loss of normal skeletal muscle atonia during REM sleep, with prominent motor activity that accompanies dream-enacting behaviors (Schenck and Mahowald, 2002). iRBD is thought to be a strong predictive factor for the development of neurodegenerative disorders, particularly those characterized by a-synuclein deposition (known as synucleinopathies), such as Parkinson's disease (PD), dementia with Lewy bodies, and multiple system atrophy (Gagnon et al., 2006; Boeve et al., 2007; Iranzo et al., 2013). Previous studies have attempted to identify biomarkers of neurodegeneration in iRBD patients to potentially predict to the development of synucleinopathies. For example, it has been reported that iRBD patients have regional changes in cerebral perfusion (Mazza 
et al., 2006; Vendette et al., 2011), brain glucose metabolic abnormalities (Wu et al., 2014; Ge et al., 2015), waking electroencephalographic impairments (Rodrigues Brazete et al., 2016), olfactory dysfunction (Fantini et al., 2006; Mahlknecht et al., 2015), and cognitive deficiencies (Fantini et al., 2011). Nevertheless, brain structural abnormalities have been poorly explored in $\mathrm{RBBD}$ patients.

Concerning the methodology, compared with hypothesis-driven region-of-interest analysis, hypothesis-free voxel-based morphometry (VBM) analysis is a fully automated, unbiased method and has been widely used to investigate neuropathological changes in neuropsychiatric illnesses and neurological disorders in vivo (Scherfler et al., 2011; Ford et al., 2013). Although recent studies have consistently demonstrated gray matter atrophy in iRBD patients (Ellmore et al., 2010; Hanyu et al., 2012; Rahayel et al., 2018), the distribution of gray matter loss was conflicting. Structural magnetic resonance imaging (MRI) investigations that were analyzed using VBM have highlighted gray matter structural alterations in unspecific and diverse regions, including the putamen, cerebellum, pons, parahippocampal gyrus, and frontal regions (Ellmore et al., 2010; Scherfler et al., 2011; Hanyu et al., 2012; Rahayel et al., 2018). Additionally, while previous studies have demonstrated region-specific perfusion or metabolic anomalies that are closely related to clinical markers in iRBD patients (Vendette et al., 2011; Ge et al., 2015), to the best of our knowledge, only one study has conducted a correlation analysis between gray matter volume (GMV) and clinical indices in this disorder (Rahayel et al., 2018). Furthermore, regional GMV in iRBD has not yet been investigated in a Chinese cohort.

This study thus aimed to investigate the GMV changes, and the relationships between GMV and clinical indices, in iRBD patients.

\section{Participants and Methods \\ Participants}

For this prospective cross-sectional study, 19 patients with iRBD (16 men and 3 women; mean age $66.6 \pm 7.0$ years) were enrolled consecutively at the Sleep and Wake Disorders Center of the Department of Neurology of Huashan Hospital, Fudan University, China. Average time between iRBD onset and MRI scanning time was $7.2 \pm 4.2$ years. All patients participating in this study fulfilled the clinical and polysomnographic (PSG) diagnosis criteria for iRBD, in accordance with the third edition of the International Classification of Sleep Disorders (Duchna, 2006; Montplaisir et al., 2010). All patients underwent a standard neurological examination and a Unified Parkinson's Disease Rating Scale motor exam (UPDRS III) (Ge et al., 2015), assessed by a blinded movement disorder specialist, and showed no overt signs of parkinsonism (UPDRS III score: $5.1 \pm 4.8$; range 0 to 19 ). None of the patients were treated with psychotropic medications. Patients showed no evidence of psychiatric disorders or dementia, and did not display any cognitive impairment according to the Mini-Mental State Examination score $(28.8 \pm 1.1$; range 27 to 30) (Crum et al., 1993). Other exclusion criteria included drug-induced RBD, sleep apnea syndrome, electroencepha- lograph (EEG) abnormalities suggestive of epilepsy, central nervous system comorbidities such as brain tumor and encephalitis, unstable hypertension, and diabetes.

A total of 20 age-matched healthy individuals, including five men and 15 women (mean age $63.7 \pm 5.9$ years), were recruited based on the China-US Biomedical Collaborative Research Program (No. 81361120393). They had no history of neurological, sleep, or psychiatric disorders, and showed no or subtle white matter lesions on brain MRI scans. These subjects took the RBD Single-Question Screen instead of PSG to rule out the existence of RBD (Nomura et al., 2011; Chahine et al., 2013).

The protocol was approved by the Ethics Committee of Huashan Hospital (approval No. KY2013-336) on January 6, 2014 (Additional file 1). All subjects gave informed consent to participate after a detailed explanation of the procedures involved.

\section{Polysomnographic evaluation}

All iRBD patients underwent one night of audio-video-PSG monitoring (Philips Electronics, Amsterdam, the Netherlands) at our sleep center laboratory. Recordings of PSG are listed as follows: EEG with six-channel scalp EEG montages (F3/F4, C3/ $\mathrm{C} 4, \mathrm{O} 1 / \mathrm{O} 2)$; bilateral electrooculography; electromyography (EMG) of mentalis and bilateral lower limbs. A nasal cannula and a mouth thermocouple were used to monitor respiration. Thoracoabdominal motion was detected by inductance plethysmogramy, and arterial oxyhemoglobin saturation $\left(\mathrm{SpO}_{2}\right)$ was continuously recorded by a cutaneous finger pulse oximeter. Sleep stages 1-4 were recorded and manually scored according to standard criteria (Montplaisir et al., 2010). REM sleep was scored based on EEG and electrooculography recordings only. The REM sleep without atonia stage was defined by the presence of excessive phasic submental electromyographic (EMG) activity during REM sleep (> 30\% of REM sleep with tonic electromyographic EMG activity or $>15 \%$ of REM sleep with phasic EMG activity) (Montplaisir et al., 2010). Audiovisual recordings were simultaneously conducted to monitor nocturnal sleep behaviors.

\section{MRI scanning}

All MRI measurements were obtained on a 3-tesla GE Discovery MR750 Scanner (Milwaukee, WI, USA) equipped with a circular polarized 8-channel head matrix coil at the Department of Radiology of Huashan Hospital of Fudan University, China. High-resolution, T1-weighted, three-dimensional anatomical brain images were acquired using an inversion recovery prepared fast spoiled gradient recalled sequence (repetition time $=11.1 \mathrm{~ms}$; echo time $=5.0 \mathrm{~ms}$; flip angle $=20^{\circ}$; matrix resolution $=256 \times 256$; voxel size $=1 \times 1 \times 1 \mathrm{~mm}^{3}$; field of view $=240 \mathrm{~mm}^{2}$; slice thickness $=1.0 \mathrm{~mm}$; 146 slices without slice gap, transverse acquisition), with the scan range from the calvarium to foramen magnum.

\section{Data preprocessing}

Image preprocessing was carried out using the VBM8 protocol (http://dbm.neuro.uni-jena.de/vbm8/) implemented within Matlab 8.3.0 (Mathworks, Inc, Sherborn, MA, USA) through 
Han XH, Li XM, Tang WJ, Yu H, Wu P, Ge JJ, Wang J, Zuo CT, Shi KY (2019) Assessing gray matter volume in patients with idiopathic rapid eye movement sleep behavior disorder. Neural Regen Res 14(5):868-875. doi:10.4103/1673-5374.249235

Statistical Parametric Mapping version 8 (SPM8, http//www. fil.ion.ucl.ac.uk/spm/software/spm8). The VBM method included several procedures. First, MRI scans from each subject were spatially normalized to the Montreal Neurological Institute/International Consortium for Brain Mapping 152 standard space template. In this step, voxel size was resampled to 3 $\times 3 \times 3 \mathrm{~mm}^{3}$. Subsequently, the normalized MRI images were segmented into gray matter, white matter, and cerebrospinal fluid. Finally, the resulting gray matter images were smoothed with a $10 \mathrm{~mm}$ full width at half maximum Gaussian kernel to increase the signal-to-noise ratio for statistical analysis.

\section{Statistical analysis}

SPM8 software (http://www.fil.ion.ucl. ac.uk/spm/software/ spm8/) was used to analyze the MRI imaging data. A two-sample $t$-test was conducted to perform voxel-to-voxel comparisons of whole-brain GMV contrasts between the two groups. In the $t$-test, sex and total intracranial volume (gray matter + white matter + cerebrospinal fluid) were added as covariates. At voxel level, the significance threshold was set at $P<0.01$ uncorrected for multiple comparisons, with an extended cluster threshold of 160 voxels. The xjView 9.6 software (http://www. alivelearn.net/xjview) was used to display brain regions with significant differences (represented with pseudo color), activation volume (cluster size), activation intensity (statistically analyzed with a t-test and expressed as $\mathrm{T}$ value; the $\mathrm{T}$ value is proportional to the intensity), Montreal Neurological Institute coordinates, and Brodmann partition (Xia et al., 2013).

To investigate the relationship between regional GMV and clinical indices in iRBD patients, we also performed a multiple regression analysis. Separate correlation analyses were run on the iRBD data using the following clinical measurements: disease duration and EMG activity. Age and total intracranial volume were added as covariates into the model to correct for the interaction between these factors. Significance levels for correlation analyses were set at $P<0.01$ uncorrected for multiple comparisons, with an extended cluster threshold of 150 voxels. The xjView 9.6 software was used to display brain regions that correlated with clinical measurements (represented with pseudo color), activation volume (cluster size), activation intensity (statistically analyzed with multiple regression analysis and expressed as $\mathrm{T}$ value; the $\mathrm{T}$ value is proportional to the intensity), Montreal Neurological Institute coordinates, and Brodmann partition.

Statistical analyses of demographic and clinical variables were conducted using the IBM SPSS Statistics19 software package (IBM Corporation, Armonk, NY, USA). Parametric independent-sample $t$-tests were used to assess differences between groups in continuous variables with normal distribution. For the nonparametric data, chi-square tests were used to determine group differences. All analyses were considered statistically significant if $P<0.05$.

\section{Results}

\section{Demographic and clinical characteristics}

Table 1 presents the demographic, PSG, and clinical characteristics of the two groups. Groups were matched for age
$(P=0.178)$. We found statistically significant differences between controls and iRBD patients in sex and total intracranial volume. Compared to controls, the percentage of men was significantly higher in iRBD patients $(P=0.000)$. In addition, the mean total intracranial volume was significantly lower in controls than in iRBD patients $(P=0.001)$. The study design is summarized in Figure $\mathbf{1}$.

Table 1 Demographic, polysomnographic, and clinical characteristics of participants

\begin{tabular}{llll}
\hline & iRBD patients & Controls & $P$-value \\
\hline Age (year) & $66.6 \pm 7.0$ & $63.7 \pm 5.9$ & $0.178^{\mathrm{a}}$ \\
Sex $(n$, male/female) & $16 / 3$ & $5 / 15$ & $<0.001^{\mathrm{b}}$ \\
TIV (mL) & $1430.7 \pm 102.4$ & $1322.7 \pm 88.5$ & $0.001^{\mathrm{a}}$ \\
UPDRS III (score) & $5.1 \pm 4.8$ & $\mathrm{NA}$ & $\mathrm{NA}$ \\
Disease duration (years) & $7.2 \pm 4.2$ & $\mathrm{NA}$ & $\mathrm{NA}$ \\
Polysomnography variables & & & \\
Total sleep duration (minute) & $331.1 \pm 43.7$ & $\mathrm{NA}$ & $\mathrm{NA}$ \\
Sleep efficiency (\%) & $74.1 \pm 10.0$ & $\mathrm{NA}$ & $\mathrm{NA}$ \\
Stage 1 NREM (\%) & $20.1 \pm 7.2$ & $\mathrm{NA}$ & $\mathrm{NA}$ \\
Stage 2 NREM (\%) & $46.8 \pm 7.6$ & $\mathrm{NA}$ & $\mathrm{NA}$ \\
REM sleep time (minute) & $59.4 \pm 19.6$ & NA & NA \\
Apnea-hypopnea index & $9.9 \pm 10.9$ & NA & NA \\
Phasic EMG activity index/chin & $20.6 \pm 11.2$ & NA & NA \\
Tonic EMG activity index/chin & $21.6 \pm 19.0$ & NA & NA \\
Phasic EMG activity index/legs & $14.2 \pm 9.3$ & NA & NA \\
Tonic EMG activity index/legs & $8.8 \pm 10.3$ & NA & NA \\
Spontaneous microarousal index & $20.2 \pm 10.3$ & NA & NA \\
Periodic limb movements in sleep & $25.1 \pm 33.5$ & NA & NA \\
\hline
\end{tabular}

${ }^{\mathrm{a}}$ Independent-sample $t$-tests; ${ }^{\mathrm{b}} \mathrm{Chi}$-square tests. iRBD: Idiopathic rapid eye movement sleep behavior disorder; TIV: total intracranial volume; UPDRS III: Unified Parkinson's Disease Rating Scale part III; REM: rapid eye movement; NREM: non-rapid eye movement; EMG: electromyograpic; NA: not available.

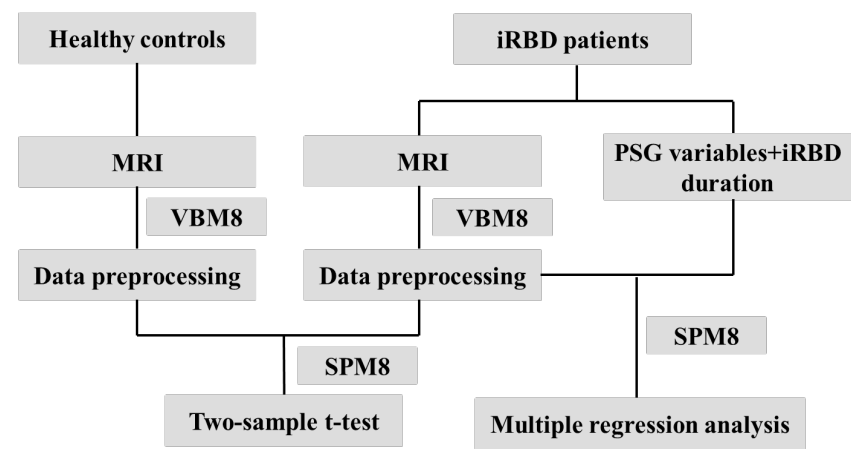

Figure 1 Study flow chart.

iRBD: Idiopathic rapid eye movement sleep behavior disorder; MRI: magnetic resonance imaging; PSG: polysomnographic; VBM: voxelbased morphometry.

\section{VBM results}

Relative to controls, iRBD patients exhibited reduced GMV in the right rolandic operculum, right postcentral gyrus, right insular lobe, right anterior cingulate gyrus, right precuneus, right posterior cingulate gyrus, left rectus gyrus, and bilateral superior frontal gyrus. Furthermore, iRBD patients displayed increased GMV in the right middle temporal gyrus and left cerebellar posterior lobe (Figure 2 and Table 2).

Multiple regression analysis demonstrated that $\mathrm{RBDD}$ du- 
Han XH, Li XM, Tang WJ, Yu H, Wu P, Ge JJ, Wang J, Zuo CT, Shi KY (2019) Assessing gray matter volume in patients with idiopathic rapid eye movement sleep behavior disorder. Neural Regen Res 14(5):868-875. doi:10.4103/1673-5374.249235

ration was positively correlated with GMV in the bilateral precuneus, bilateral calcarine fissure and surrounding cortex, bilateral cerebellar posterior lobe, right cuneus, right superior parietal gyrus, left postcentral gyrus, left posterior cingulate gyrus, left hippocampus, left lingual gyrus, left parahippocampal gyrus, left middle occipital gyrus, and left middle temporal gyrus. Furthermore, phasic chin EMG activity was positively correlated with GMV in the bilateral hippocampus, bilateral parahippocampal gyrus, bilateral precuneus, bilateral cerebellar posterior lobe, bilateral fusiform gyrus, right precentral gyrus, right superior frontal gyrus, right cuneus, right inferior parietal lobule, right angular gyrus, right superior parietal gyrus, right paracentral lobule, and left calcarine fissure and surrounding cortex (Figures 3, 4 and Table 3).

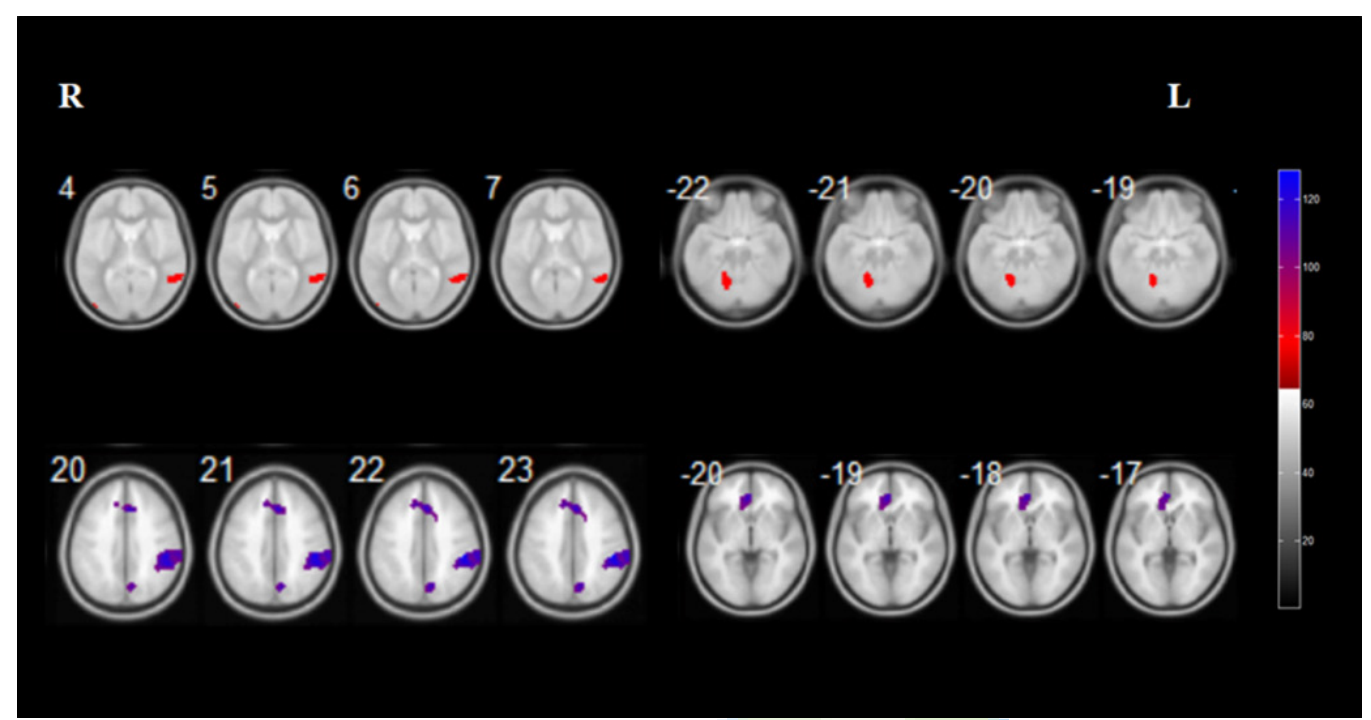

Figure 2 Brain regions with relatively increased (red) and decreased (purple) gray matter volume in patients with idiopathic rapid eye movement sleep behavior disorder.

Results of statistical analysis are superimposed onto the axial template brain mapping in the Montreal Neurological Institute standard coordinate system. Corresponding anatomical locations of color regions can be found in Table 2 .

Table 2 Brain regions with significant gray matter volume differences between iRBD patients and controls

\begin{tabular}{|c|c|c|c|c|c|c|}
\hline \multirow[b]{2}{*}{ Regions } & \multirow[b]{2}{*}{$\mathrm{BA}$} & \multirow[b]{2}{*}{ Cluster size (voxels) } & \multirow[b]{2}{*}{ Peak intensity } & \multicolumn{3}{|c|}{ Peak coordinates $(\mathrm{mm})$} \\
\hline & & & & $x$ & $y$ & $z$ \\
\hline \multicolumn{7}{|l|}{ Increased gray matter volume } \\
\hline Right middle temporal gyrus & 21 & 301 & 3.52 & 55 & -49 & 6 \\
\hline Left cerebelum_4_5; Left cerebelum_6 & I & 744 & 3.28 & -15 & -60 & -28 \\
\hline \multicolumn{7}{|l|}{ Decreased gray matter volume } \\
\hline Right Rolandic operculum; Right postcentral gyrus; Right insular lobe & 13,43 & 1286 & 4.01 & 48 & -13 & 23 \\
\hline Right anterior cingulate gyrus; Left superior frontal gyrus & 9 & 544 & 3.53 & 3 & 44 & 21 \\
\hline Right precuneus; Right posterior cingulate gyrus & 31 & 462 & 3.18 & 7 & -51 & 23 \\
\hline Left rectus gyrus; Left superior frontal gyrus & 11 & 415 & 3.64 & -3 & 56 & -25 \\
\hline
\end{tabular}

iRBD: Idiopathic rapid eye movement sleep behavior disorder; BA: Brodmann area. Two-sample $t$-test, $P<0.01$ uncorrected for multiple comparisons.

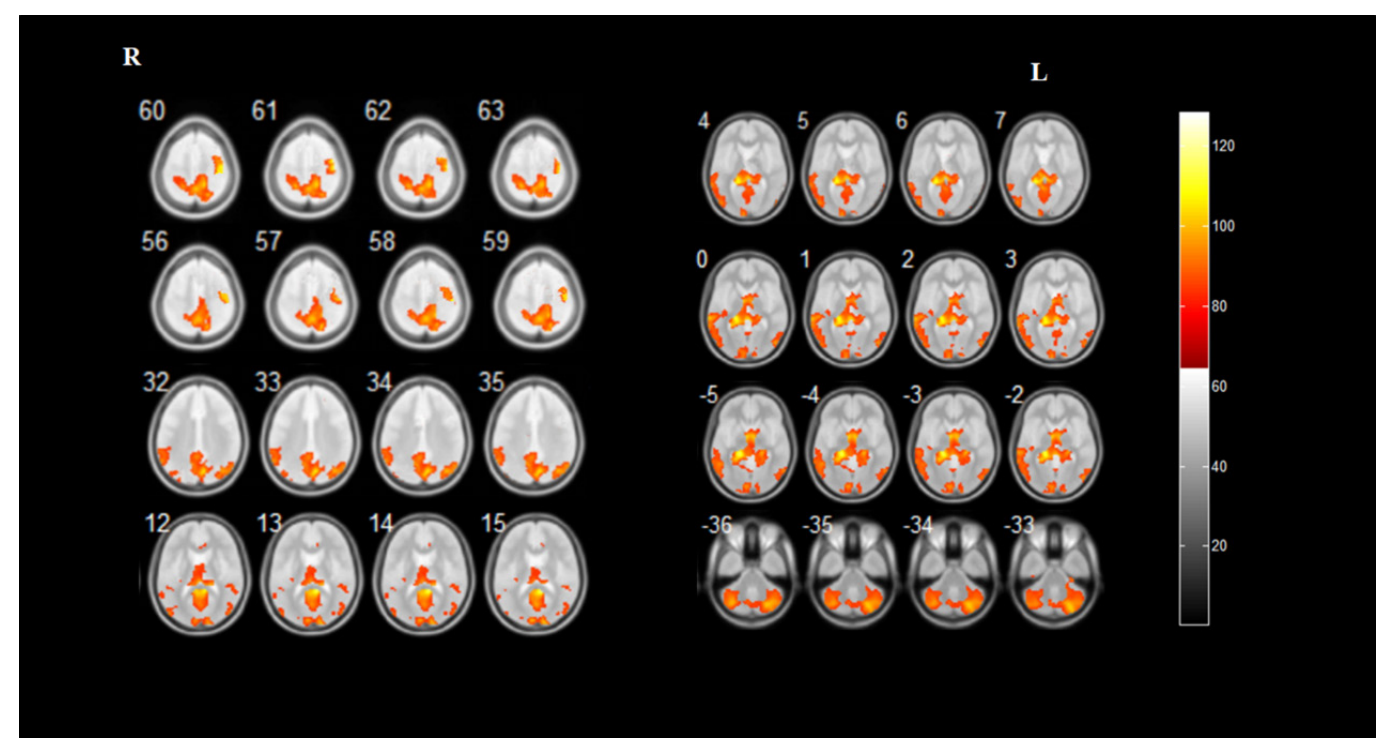

Figure 3 Brain regions correlate with the duration of idiopathic rapid eye movement sleep behavior disorder. Results of statistical analysis are superimposed on the axial template brain mapping in the Montreal Neurological Institute standard coordinate system. Corresponding anatomical locations of color regions can be found in Table 3 . 
Han XH, Li XM, Tang WJ, Yu H, Wu P, Ge JJ, Wang J, Zuo CT, Shi KY (2019) Assessing gray matter volume in patients with idiopathic rapid eye movement sleep behavior disorder. Neural Regen Res 14(5):868-875. doi:10.4103/1673-5374.249235

Table 3 Anatomical locations of regions with significant clinical correlation in iRBD patients

\begin{tabular}{|c|c|c|c|c|c|c|}
\hline \multirow[b]{2}{*}{ Regions } & \multirow[b]{2}{*}{ BA } & \multirow[b]{2}{*}{ Cluster size (voxels) } & \multirow[b]{2}{*}{ Peak intensity } & \multicolumn{3}{|c|}{ Peak coordinates $(\mathrm{mm})$} \\
\hline & & & & $x$ & $y$ & $z$ \\
\hline \multicolumn{7}{|l|}{ Duration } \\
\hline \multicolumn{7}{|l|}{ Positive } \\
\hline Bilateral precuneus; bilateral calcarine fissure and surrounding cortex & 23,30 & 50148 & 6.73 & 9 & -45 & 15 \\
\hline Right cuneus & 17 & & 4.99 & 15 & -96 & 14 \\
\hline Left postcentral gyrus; bilateral precuneus; right superior parietal gyrus & 5,7 & & 5.32 & 15 & -45 & 60 \\
\hline Left posterior cingulate gyrus; left precuneus & 29 & & 5.24 & -3 & -49 & 9 \\
\hline Left posterior cingulate gyrus; left precuneus; left hippocampus & 27,29 & & 6.22 & -17 & -40 & 7 \\
\hline Left hippocampus; left lingual gyrus; left parahippocampal gyrus & 27,19 & & 6.24 & -18 & -34 & -6 \\
\hline Left middle occipital gyrus & 19 & & 5.67 & -33 & -70 & 33 \\
\hline Left postcentral gyrus; bilateral precuneus & 7,31 & & 5.28 & 8 & -72 & 33 \\
\hline Right superior parietal gyrus; bilateral precuneus & 5,7 & & 5.27 & 15 & -67 & 57 \\
\hline Left middle temporal gyrus & 21,22 & & 5.09 & -68 & -33 & 1 \\
\hline Left cerebellum_crus2 & / & & 5.18 & -39 & -66 & -39 \\
\hline Right cerebellum_crus1 & I & & 5.65 & 30 & -79 & -33 \\
\hline \multicolumn{7}{|l|}{ Phasic chin EMG activity } \\
\hline \multicolumn{7}{|l|}{ Positive } \\
\hline Right hippocampus; right fusiform gyrus; right parahippocampal gyrus & 30,36 & 45897 & 6.50 & 23 & -24 & -15 \\
\hline Right hippocampus; right parahippocampal gyrus & 28 & & 5.96 & 29 & -19 & -11 \\
\hline Left hippocampus; left fusiform gyrus; left parahippocampal gyrus & 37 & & 6.06 & -27 & -43 & -12 \\
\hline Right precentral gyrus; right superior frontal gyrus & 4,6 & & 6.29 & 44 & -16 & 60 \\
\hline Right cuneus; bilateral precuneus & 7 & & 5.79 & 8 & -72 & 33 \\
\hline Right inferior parietal lobule; right angular gyrus & 39,40 & & 5.54 & 41 & -63 & 45 \\
\hline Right precentral gyrus & 6 & & 5.60 & 44 & 17 & 46 \\
\hline Right superior parietal gyrus & 7 & & 5.33 & 17 & -63 & 57 \\
\hline Bilateral precuneus; right paracentral lobule & 5 & & 5.59 & 15 & -45 & 60 \\
\hline Bilateral precuneus; left calcarine fissure and surrounding cortex & 31,30 & & 5.26 & 0 & -66 & 19 \\
\hline Right cerebellum_crus1 & l & & 5.32 & 29 & -79 & -32 \\
\hline Left cerebellum_crus2 & I & & 5.74 & -39 & -63 & -41 \\
\hline
\end{tabular}

iRBD: Idiopathic rapid eye movement sleep behavior disorder; BA: Brodmann area; EMG: electromyographic. Multiple regression analysis, $P<0.01$ uncorrected for multiple comparisons.

\section{Discussion}

In this article, we used VBM to investigate structural differences between the brains of healthy controls and iRBD patients. Additionally, using SPM analysis we also identified several brain regions that were volumetrically correlated with clinical measurements in iRBD patients.

A limited number of structural MRI studies have reported contradictory results in patients with iRBD (Ellmore et al., 2010; Scherfler et al., 2011; Hanyu et al., 2012; Rahayel et al., 2015), and gray matter changes are not entirely understood in this disorder. To further clarify the neuropathology from an anatomical viewpoint, we used VBM analysis to identify any regions with GMV changes in $\mathrm{iRBD}$. The distribution of GMV reduction in the frontal and cingulate gyri in our iRBD population coincided with the atrophic regions previously described by Rahayel et al. (2015, 2018). Indeed, hypoperfusion and hypometabolism in the two regions has also been observed in iRBD patients using single photon emission computed tomography (Vendette et al., 2011, 2012) and positron emission tomography (Fujishiro et al., 2010; Ota et al., 2016). In addition, a study that performed quantitative analyses of waking EEG activity demonstrated considerably slower activity in the frontal regions of iRBD patients' brains (Rodrigues Brazete et al., 2016). Nevertheless, with uncertainty remaining, further research is needed to identify the exact relationships between structural and functional alterations in iRBD patients.

In contrast to earlier volumetric studies of iRBD, we detected no significant GMV reduction in the cerebellum, pons, parahippocampal gyrus (Hanyu et al., 2012), or putamen (Ellmore et al., 2010), nor a GMV increase in the hippocampus (Scherfler et al., 2011) of iRBD patients. These disparities might reflect methodological differences, which include sample size and patient characteristics, as well as image analysis approaches.

Of note, ours was a novel finding that iRBD patients had reduced GMV in the insular lobe, cingulate gyrus, postcentral gyrus, and rectus gyrus. Previously, despite lacking significance, a subtle GMV reduction in the insular lobe was observed in PD patients with probable RBD compared to those without probable RBD (Ford et al., 2013). Similarly, studies reported that $\mathrm{PD}$ patients with probable RBD exhibited diminished GMV in the cingulate gyrus (Boucetta et al., 2016; 
Han XH, Li XM, Tang WJ, Yu H, Wu P, Ge JJ, Wang J, Zuo CT, Shi KY (2019) Assessing gray matter volume in patients with idiopathic rapid eye movement sleep behavior disorder. Neural Regen Res 14(5):868-875. doi:10.4103/1673-5374.249235

Lim et al., 2016). It could be speculated that the cingulate gyrus and insular lobe are strongly associated with RBD per se, irrespective of its existence as an idiopathic disease or a secondary disease associated with synucleinopathies. Similar to the results from iRBD patients in our study, a previous study found that patients with prodromal dementia with Lewy bodies had diminished GMV in the insular lobe and cingulate cortex (Blanc et al., 2016), which may suggest a potential connection between iRBD and dementia with Lewy bodies.

According to the widely acknowledged neuropathological staging proposed by Braak et al. (2003), the neurodegeneration responsible for RBD symptoms occurs early on, in the brain stem. The neurodegeneration then extends to the midbrain, where it gives rise to the motor symptoms of PD, before spreading to limbic and neocortical areas. However, our study demonstrated that the occurrence of structural neocortical involvement precedes mesencephalic region abnormalities, at least in iRBD. There are several possible interpretations for these discrepancies. First, in PD patients only slight midbrain atrophy has been detected, and there is no correlation between midbrain atrophy and striatal dopamine function (Makinen et al., 2016). As a potential precursor of $\mathrm{PD}, \mathrm{iRBD}$ is thus believed to present mild midbrain atrophy or even no atrophy. Second, from a clinicopathological perspective, tau burden is closely associated with midbrain atrophy. In particular, the density of tau-positive structures, such as neurofibrillary tangles and glial fibrillary tangles, is reported to be strongly linked to the severity of midbrain atrophy (Aiba et al., 1997). As previously mentioned, iRBD appears to be a precursor for synucleinopathies, and this may account for the absence of atrophy in the midbrain in iRBD. Third, such a phenomenon would also be supported by the observation that cortical Alzheimer-type and a-synuclein pathological aggregation concurrently occur in a significant proportion of PD and dementia with Lewy bodies cases (Hurtig et al., 2000; Harding and Halliday, 2001; Pletnikova et al., 2005; Jellinger, 2012). It is tempting to suggest that this provides additional evidence that iRBD might have an analogous pathological feature. Nonetheless, pathophysiological substrates related to the GMV modifications observed in our iRBD patients need to be further investigated.

Besides GMV alterations in iRBD patients, we also detected correlations between structural changes and clinical measurements. It is of note that the regression analysis yielded exclusively positive correlations, either for iRBD duration or for phasic chin EMG activity, although this relationship is still unclear. However, we observed that mean total intracranial volume in our iRBD patients was significantly higher than in controls, and that similar neurofunctional correlations have been previously reported in $\mathrm{RBD}$ populations. Whether drug therapy affects GMV and contributes to this finding in iRBD patients is unknown, and needs further investigation. The positive correlation between iRBD duration and GMV in the posterior cingulate gyrus is supported by previous research that demonstrated corresponding correlations of regional metabolism and perfusion with iRBD du- ration and deficient color vision discrimination, separately (Vendette et al., 2011; Ge et al., 2015). Likewise, perfusion in the cerebellar posterior lobe has been reported to positively correlate with a deficit in olfaction and color vision in iRBD patients (Vendette et al., 2011), which our findings also supported. Further studies applying multimodal neuroimaging techniques should be conducted to explore the interaction between metabolism/perfusion and anatomy.

Although previous studies have demonstrated increased perfusion, metabolism, or gray matter density in the hippocampus and parahippocampal gyrus (Scherfler et al., 2011; Vendette et al., 2011; Ge et al., 2015), prompting the neuronal reorganization of the hippocampal region in $\mathrm{RBD}$, no significant GMV modifications in this area were found in the current study. One potential reason for the absence of a GMV increase in this region might be the relatively short iRBD duration of our patients. However, we did observe that phasic chin EMG activity positively correlated with GMV in the hippocampal region. There are two possible interpretations for this result. First, earlier studies observed positive correlations between perfusion/metabolism in the hippocampus/parahippocampal gyrus, and phasic chin EMG activity, motor signs, olfactory disturbances, and color discrimination deficiencies in iRBD patients (Vendette et al., 2011; Dang-Vu et al., 2012; Ge et al., 2015). The positive correlation observed in the present study might then reinforce the concept that the hippocampus/parahippocampal gyrus is a critical component in the neuropathology of iRBD. Collectively, discoveries in the hippocampal regions validate the involvement of the limbic system, and support the idea of iRBD as a contributing factor for cognitive impairment, although this remains to be further investigated. In addition, the severity of REM atonia loss at baseline can predict imminent parkinsonism in iRBD patients (Postuma et al., 2010), and elevated hippocampal perfusion at baseline has been proposed to signal the neurodegeneration that converts RBD into synucleinopathies (Dang-Vu et al., 2012). Coupled with our findings, we speculate that GMV modifications in the hippocampal region may thus be a promising predictive imaging biomarker of impending conversion from iRBD. However, the precision of this prediction must be viewed with caution given our relatively limited sample size, and needs to be verified by a longer period of follow up in a larger iRBD cohort.

Despite adding possible confounding factors into the multiple regression design, such as age and total intracranial volume, we identified that brain regions correlating with the two clinical indices overlapped, such as in the bilateral cerebellar posterior lobe. One possible explanation might be that excessive tonic and phasic EMG activity during REM sleep increases over time in iRBD patients (Iranzo et al., 2009). Our generated overlapping area could therefore be a consequence of the synchronization of underpinning neuropathophysiological substrates of the two clinical indices.

There are some limitations in this study. First, the male to female ratio was discordant between the two groups. Considering that there are subtle differences in brain structures in healthy men and women (Cosgrove et al., 2007), we can- 


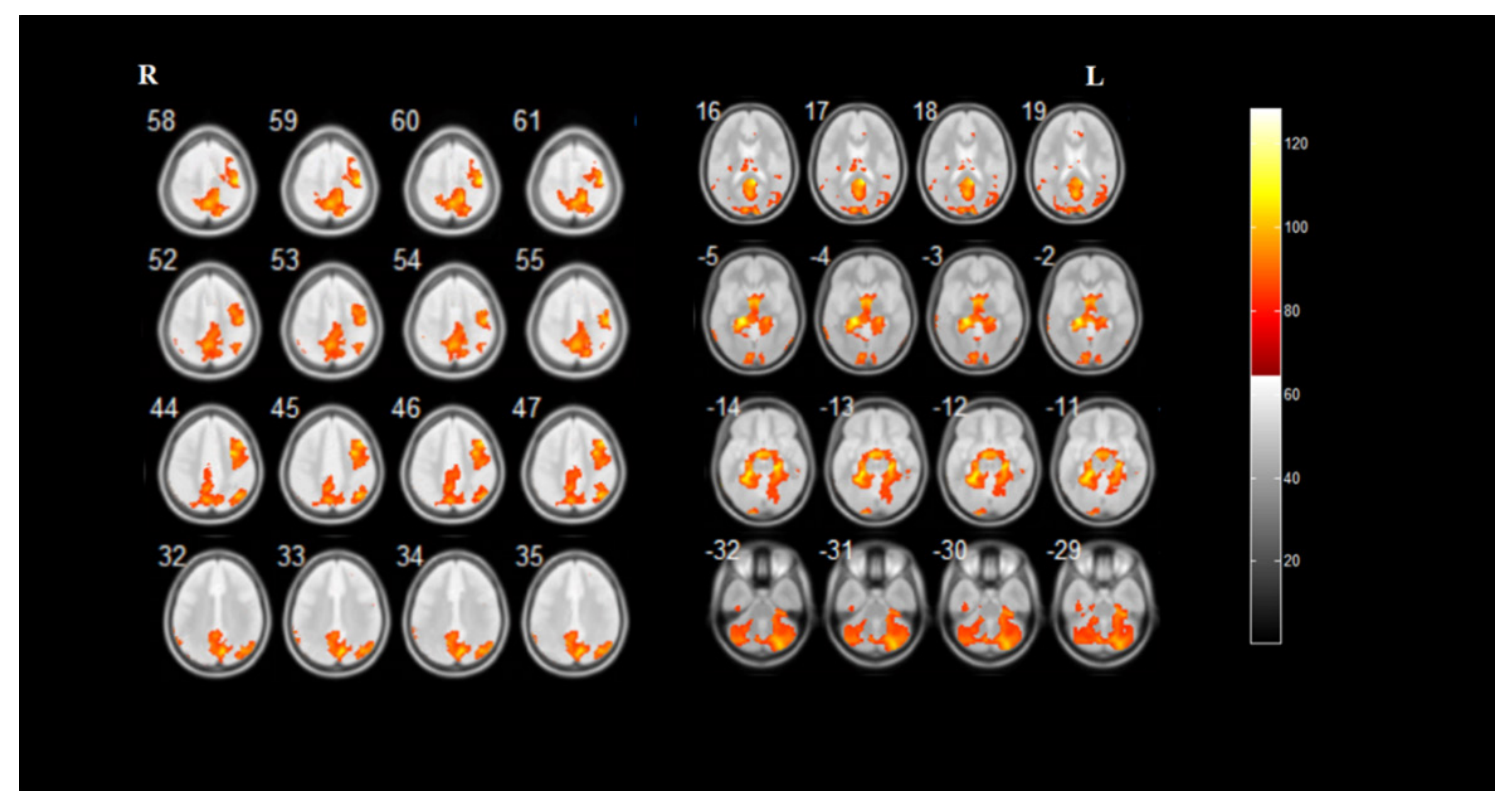

Figure 4 Brain regions correlate with phasic chin electromyographic activity in patients with idiopathic rapid eye movement sleep behavior disorder.

Results of statistical analysis are superimposed on the axial template brain mapping in the Montreal Neurological Institute standard coordinate system. Corresponding anatomical locations of color regions can be found in Table 3.

not rule out the possibility that this might have affected our results. Second, control subjects were enrolled simply based on a questionnaire and without validation of the diagnosis with PSG. Third, although VBM has been extensively used to detect gray matter loss, it has been proposed that VBM has inherent drawbacks (Davatzikos, 2004; Ioannidis, 2011), such as a tendency to significantly bias results towards group differences that are highly localized in space and linear nature. Finally, we had a relatively small sample size and the subjects were recruited from a single organization, which means that the participants were not a typical sample of the Chinese population. Thus, large-scale, multi-center research should be conducted using validated PSG-tested subjects, ideally in a prospective way.

In summary, we described brain GMV changes in $\mathrm{RBBD}$ patients using VBM. We also presented correlations between regional GMV and iRBD duration and phasic chin EMG activity, which primarily highlighted a limbic involvement. Our findings provide anatomical bases for previously observed disturbances in cerebral function and clinical manifestations in this population. Future longitudinal studies in our iRBD cohort are needed to clarify the eventual outcomes of our iRBD patients, and to elucidate whether specific gray matter abnormalities could predict progression towards synucleinopathies.

Author contributions: Study design and concept: CTZ and XHH. Experiment implement and data acquisition: WJT, HY, JJG, JW, PW and KYS. Data analysis: XHH and XML. Paper writing: XHH. Study supervision and paper revision: CTZ. All authors approved the final version of the paper.

Conflicts of interest: None declared.

Financial support: This study was supported by the China-US Biomedical Collaborative Research Program, No. 81361120393 (to CTZ); the National Natural Science Foundation of China, No. 81401135 (to PW), 81671239 (to CTZ); and Shanghai Sailing Program, No. 18YF1403100 (to JJG). The funding bodies played no role in the study design, in the collection, analysis and in- terpretation of data, in the writing of the paper, and in the decision to submit the paper for publication.

Institutional review board statement: Approval for the study was obtained from the Ethics Committee of Huashan Hospital, China (approval number: KY2013-336) on Januray 6, 2014. All participants provided written informed consent for their inclusion in the present study.

Declaration of participant consent: The authors certify that they have obtained all appropriate participant consent forms. In the form the participants have given their consent for their images and other clinical information to be reported in the journal. The participants understand that their names and initials will not be published and due efforts will be made to conceal their identity, but anonymity cannot be guaranteed.

Reporting statement: This study followed the STrengthening the Reporting of OBservational studies in Epidemiology (STROBE) statement.

Biostatistics statement: The statistical methods of this study were reviewed by the biostatistician of Fudan University in China.

Copyright license agreement: The Copyright License Agreement has been signed by all authors before publication.

Data sharing statement: Individual participant data will be available (including data dictionaries). All of the individual participant data collected during the trial, after deidentification. Study protocol, statistical analysis plan and informed consent form will be available. Data will be available immediately following publication, no end date. Data are available indefinitely at http://www.nrronline.org.

Plagiarism check: Checked twice by iThenticate.

Peer review: Externally peer reviewed.

Open access statement: This is an open access journal, and articles are distributed under the terms of the Creative Commons Attribution-NonCommercial-ShareAlike 4.0 License, which allows others to remix, tweak, and build upon the work non-commercially, as long as appropriate credit is given and the new creations are licensed under the identical terms.

Open peer reviewers: Kazunori Adachi, Meikai University School of Dentistry, Japan; L Cocito, Universita degli Studi di Genova, Italy.

Additional file:

Additional file 1: Report of Ethics Committee (Chinese).

Additional file 2: Open peer review reports 1 and 2.

\section{References}

Aiba I, Hashizume Y, Yoshida M, Okuda S, Murakami N, Ujihira N (1997) Relationship between brainstem MRI and pathological findings in progressive supranuclear palsy--study in autopsy cases. J Neurol Sci 152:210-217.

Blanc F, Colloby SJ, Cretin B, de Sousa PL, Demuynck C, O’Brien JT, Martin-Hunyadi C, McKeith I, Philippi N, Taylor JP (2016) Grey matter atrophy in prodromal stage of dementia with Lewy bodies and Alzheimer's disease. Alzheimers Res Ther 8:31. 
Han XH, Li XM, Tang WJ, Yu H, Wu P, Ge JJ, Wang J, Zuo CT, Shi KY (2019) Assessing gray matter volume in patients with idiopathic rapid eye movement sleep behavior disorder. Neural Regen Res 14(5):868-875. doi:10.4103/1673-5374.249235

Boeve BF, Silber MH, Saper CB, Ferman TJ, Dickson DW, Parisi JE, Benarroch EE, Ahlskog JE, Smith GE, Caselli RC, Tippman-Peikert M, Olson EJ, Lin SC, Young T, Wszolek Z, Schenck CH, Mahowald MW, Castillo PR, Del Tredici K, Braak H (2007) Pathophysiology of REM sleep behaviour disorder and relevance to neurodegenerative disease. Brain 130:2770-2788.

Boucetta S, Salimi A, Dadar M, Jones BE, Collins DL, Dang-Vu TT (2016) Structural brain alterations associated with rapid eye movement sleep behavior disorder in Parkinson's disease. Sci Rep 6:26782.

Braak H, Del Tredici K, Rub U, de Vos RA, Jansen Steur EN, Braak E (2003) Staging of brain pathology related to sporadic Parkinson's disease. Neurobiol Aging 24:197-211.

Chahine LM, Daley J, Horn S, Colcher A, Hurtig H, Cantor C, Dahodwala N (2013) Questionnaire-based diagnosis of REM sleep behavior disorder in Parkinson's disease. Mov Disord 28:1146-1149.

Cosgrove KP, Mazure CM, Staley JK (2007) Evolving knowledge of sex differences in brain structure, function, and chemistry. Biol Psychiatry 62:847-855.

Crum RM, Anthony JC, Bassett SS, Folstein MF (1993) Population-based norms for the Mini-Mental State Examination by age and educational level. JAMA 269:2386-2391.

Dang-Vu TT, Gagnon JF, Vendette M, Soucy JP, Postuma RB, Montplaisir J (2012) Hippocampal perfusion predicts impending neurodegeneration in REM sleep behavior disorder. Neurology 79:2302-2306.

Davatzikos C (2004) Why voxel-based morphometric analysis should be used with great caution when characterizing group differences. Neuroimage 23:17-20.

Duchna HW (2006) Sleep-related breathing disorders--a second edition of the International Classification of Sleep Disorders (ICSD-2) of the American Academy of Sleep Medicine (AASM). Pneumologie 60:568-575.

Ellmore TM, Hood AJ, Castriotta RJ, Stimming EF, Bick RJ, Schiess MC (2010) Reduced volume of the putamen in REM sleep behavior disorder patients. Parkinsonism Relat Disord 16:645-649.

Fantini ML, Postuma RB, Montplaisir J, Ferini-Strambi L (2006) Olfactory deficit in idiopathic rapid eye movements sleep behavior disorder. Brain Res Bull 70:386-390.

Fantini ML, Farini E, Ortelli P, Zucconi M, Manconi M, Cappa S, Ferini-Strambi L (2011) Longitudinal study of cognitive function in idiopathic REM sleep behavior disorder. Sleep 34:619-625.

Ford AH, Duncan GW, Firbank MJ, Yarnall AJ, Khoo TK, Burn DJ, O’Brien JT (2013) Rapid eye movement sleep behavior disorder in Parkinson's disease: magnetic resonance imaging study. Mov Disord 28:832-836.

Fujishiro H, Iseki E, Murayama N, Yamamoto R, Higashi S, Kasanuki K, Suzuki M, Arai H, Sato K (2010) Diffuse occipital hypometabolism on [18 F]-FDG PET scans in patients with idiopathic REM sleep behavior disorder: prodromal dementia with Lewy bodies? Psychogeriatrics 10:144-152.

Gagnon JF, Postuma RB, Mazza S, Doyon J, Montplaisir J (2006) Rapid-eye-movement sleep behaviour disorder and neurodegenerative diseases. Lancet Neurol 5:424-432.

Ge J, Wu P, Peng S, Yu H, Zhang H, Guan Y, Eidelberg D, Zuo C, Ma Y, Wang J (2015) Assessing cerebral glucose metabolism in patients with idiopathic rapid eye movement sleep behavior disorder. J Cereb Blood Flow Metab 35:1902.

Hanyu H, Inoue Y, Sakurai H, Kanetaka H, Nakamura M, Miyamoto T, Sasai T, Iwamoto T (2012) Voxel-based magnetic resonance imaging study of structural brain changes in patients with idiopathic REM sleep behavior disorder. Parkinsonism Relat Disord 18:136-139.

Harding AJ, Halliday GM (2001) Cortical Lewy body pathology in the diagnosis of dementia. Acta Neuropathol 102:355-363.

Hurtig HI, Trojanowski JQ, Galvin J, Ewbank D, Schmidt ML, Lee VM, Clark CM, Glosser G, Stern MB, Gollomp SM, Arnold SE (2000) Alpha-synuclein cortical Lewy bodies correlate with dementia in Parkinson's disease. Neurology 54:1916-1921.

Ioannidis JP (2011) Excess significance bias in the literature on brain volume abnormalities. Arch Gen Psychiatry 68:773-780.

Iranzo A, Ratti PL, Casanova-Molla J, Serradell M, Vilaseca I, Santamaria J (2009) Excessive muscle activity increases over time in idiopathic REM sleep behavior disorder. Sleep 32:1149-1153.

Iranzo A, Tolosa E, Gelpi E, Molinuevo JL, Valldeoriola F, Serradell M, Sanchez-Valle R, Vilaseca I, Lomena F, Vilas D, Llado A, Gaig C, Santamaria J (2013) Neurodegenerative disease status and post-mortem pathology in idiopathic rapid-eye-movement sleep behaviour disorder: an observational cohort study. Lancet Neurol 12:443-453.
Jellinger KA (2012) Neuropathology of sporadic Parkinson's disease: evaluation and changes of concepts. Mov Disord 27:8-30.

Lim JS, Shin SA, Lee JY, Nam H, Lee JY, Kim YK (2016) Neural substrates of rapid eye movement sleep behavior disorder in Parkinson's disease. Parkinsonism Relat Disord 23:31-36.

Mahlknecht P, Iranzo A, Hogl B, Frauscher B, Muller C, Santamaria J, Tolosa E, Serradell M, Mitterling T, Gschliesser V, Goebel G, Brugger F, Scherfler C, Poewe W, Seppi K, Sleep Innsbruck Barcelona G (2015) Olfactory dysfunction predicts early transition to a Lewy body disease in idiopathic RBD. Neurology 84:654-658.

Makinen E, Joutsa J, Isotalo J, Kaasinen V (2016) No relevant midbrain atrophy in Parkinson's disease. Acta Neurol Scand 134:378-381.

Mazza S, Soucy JP, Gravel P, Michaud M, Postuma R, Massicotte-Marquez J, Decary A, Montplaisir J (2006) Assessing whole brain perfusion changes in patients with REM sleep behavior disorder. Neurology 67:1618-1622.

Montplaisir J, Gagnon JF, Fantini ML, Postuma RB, Dauvilliers Y, Desautels A, Rompre S, Paquet J (2010) Polysomnographic diagnosis of idiopathic REM sleep behavior disorder. Mov Disord 25:2044-2051.

Nomura T, Inoue Y, Kagimura T, Uemura Y, Nakashima K (2011) Utility of the REM sleep behavior disorder screening questionnaire (RBDSQ) in Parkinson's disease patients. Sleep Med 12:711-713.

Ota K, Fujishiro H, Kasanuki K, Kondo D, Chiba Y, Murayama N, Arai H, Sato K, Iseki E (2016) Prediction of later clinical course by a specific glucose metabolic pattern in non-demented patients with probable REM sleep behavior disorder admitted to a memory clinic: A case study. Psychiatry Res 248:151-158.

Pletnikova O, West N, Lee MK, Rudow GL, Skolasky RL, Dawson TM, Marsh L, Troncoso JC (2005) Abeta deposition is associated with enhanced cortical alpha-synuclein lesions in Lewy body diseases. Neurobiol Aging 26:1183-1192.

Postuma RB, Gagnon JF, Rompre S, Montplaisir JY (2010) Severity of REM atonia loss in idiopathic REM sleep behavior disorder predicts Parkinson disease. Neurology 74:239-244.

Rahayel S, Postuma RB, Montplaisir J, Bedetti C, Brambati S, Carrier J, Monchi O, Bourgouin PA, Gaubert M, Gagnon JF (2018) Abnormal gray matter shape, thickness, and volume in the motor cortico-subcortical loop in idiopathic rapid eye movement sleep behavior disorder: association with clinical and motor features. Cereb Cortex 28:658-671.

Rahayel S, Montplaisir J, Monchi O, Bedetti C, Postuma RB, Brambati S, Carrier J, Joubert S, Latreille V, Jubault T, Gagnon JF (2015) Patterns of cortical thinning in idiopathic rapid eye movemenst sleep behavior disorder. Mov Disord 30:680-687.

Rodrigues Brazete J, Gagnon JF, Postuma RB, Bertrand JA, Petit D, Montplaisir J (2016) Electroencephalogram slowing predicts neurodegeneration in rapid eye movement sleep behavior disorder. Neurobiol Aging 37:74-81.

Schenck CH, Mahowald MW (2002) REM sleep behavior disorder: clinical, developmental, and neuroscience perspectives 16 years after its formal identification in SLEEP. Sleep 25:120-138.

Scherfler C, Frauscher B, Schocke M, Iranzo A, Gschliesser V, Seppi K, Santamaria J, Tolosa E, Hogl B, Poewe W, Group S (2011) White and gray matter abnormalities in idiopathic rapid eye movement sleep behavior disorder: a diffusion-tensor imaging and voxel-based morphometry study. Ann Neurol 69:400-407.

Vendtte M, Montplaisir J, Gosselin N, Soucy JP, Postuma RB, Dang-Vu TT, Gagnon JF (2012) Brain perfusion anomalies in rapid eye movement sleep behavior disorder with mild cognitive impairment. Mov Disord 27:1255-1261.

Vendette M, Gagnon JF, Soucy JP, Gosselin N, Postuma RB, Tuineag M, Godin I, Montplaisir J (2011) Brain perfusion and markers of neurodegeneration in rapid eye movement sleep behavior disorder. Mov Disord 26:1717-1724.

Wu P, Yu H, Peng S, Dauvilliers Y, Wang J, Ge J, Zhang H, Eidelberg D, Ma Y, Zuo C (2014) Consistent abnormalities in metabolic network activity in idiopathic rapid eye movement sleep behaviour disorder. Brain 137:3122-3128.

Xia J, Miu J, Ding H, Wang X, Chen H, Wang J, Wu J, Zhao J, Huang H, Tian W (2013) Changes of brain gray matter structure in Parkinson's disease patients with dementia. Neural Regen Res 8:1276-1285.

P-Reviewers: Adachi K, Cocito L; C-Editor: Zhao M; S-Editor: $\mathrm{Li} \mathrm{CH}$; L-Editors: Gardner B, Wysong S, Qiu Y, Song LP; T-Editor: Liu XL 